\title{
A Brief Analysis of the Sentiment of "Transcedent" in Painting
}

\author{
Yanli $\mathrm{Yu}$ \\ Department of Fine Arts, Silk Road Academy of Arts, Weinan Normal University, Weinan, Shaanxi, \\ 714099
}

Keywords: Imagery painting; Transcedent; Traditional culture; Return to nature

\begin{abstract}
Imagery painting is an emerging art form that combines western painting materials with Chinese traditional humanistic ideas and philosophy. It is more or less influenced by Western modern art, and combines the concepts of "spirit", charm" and "feeling for scenery" inherited from traditional Chinese paintings, which truly achieves the localization of paintings in China. Imagery painting and literary freehand painting have their roots in the same strain, adhering to Taoist ideologies of "being back to nature" and "returning to nature" which present unique and extraordinary temperament, that is, the temperament of "transcedent". This paper briefly discusses the meaning and development background of imagery paintings, the basic meaning of "transcedent" and the traditional philosophical view that they infiltrate, and discusses the imagery paintings from the three perspectives of the image effect, the choice of theme, and the picture quality of imagery. The specific artistic works are taken as examples to discuss the theme. Imagery paintings do not only break the old methods of creation, but also become a bridge to link to the traditional culture. It distinguishes itself from western local paintings in terms of pure painting language and humanistic feelings, and occupies an important position in modern and contemporary Chinese paintings.
\end{abstract}

\section{The Occurrence and Evolution of Imagery Paintings}

As an important breakthrough in the development of Chinese modern art, the occurrence and evolution of imagery painting is undoubtedly a great art revolution. Taking $\mathrm{Wu}$ Guanzhong and other painters who were the first batch of painters who stayed in France as examples, they brought French modernism back to China around the middle of the 20th century, and conducted a cultural and material blending with Chinese traditional art concepts. Most of the painters of Europe in the 20th century have come out of the gloom and color of realism and impressionism of the past century, and began to explore more pure points, lines, faces and the collision of painting languages in form or color. However, judging from the current state of Chinese art, Chinese imagery paintings seem to have long been close to the completion of the exploration of abstract art, and is facing great challenges in the form of new art. In Xie He's "six laws" of South Qi Dynasty, he first mentioned "vivid rhyme" and "brushwork of bone method". The concept of "imaging" conveyed in "six laws" is closely related to traditional philosophical concepts, and it has undergone verification in different eras, which lasted for a very long time in the history of Chinese paintings. The appearance and development of freehand paintings has brought this concept to its peak. It is still widely circulated today. It can be seen that Chinese art has always been steadfast in its pursuit of "imaging".

The appearance of imagery paintings undoubtedly achieved the perfect combination of the traditional Chinese art concept and Western painting materials to a certain extent, which did not only break the solid and modeling expression method of paintings, but also broke through the use of single ink materials. In the process of creation, imagery paintings focus on refining the intuition of the object and attaining a state of "inner and out-of-mind" and "being united with everything", which is the reason that imagery paintings are not limited to the objective reproduction of the image. However, in the creation, we take the initiative to summarize and choose, and combine the most direct impressions and perceptions of the subject of creation with natural objects, and make it a kind of sculptural image-making, thus demonstrating the traditional Chinese philosophy and humanistic spirit.

In Chinese painting, the painter's process of viewing objects is actually a process of integrating personal feelings into nature and realizing the blending of things. Here, the painter's feelings about 
the nature and the universe are images that saturate the subjective consciousness of the painter. They are very much emphasizing the grasp of the momentum and charm of objective images, which is consistent with the painter's personal feelings and psychological logic. It is not only the traditional literati thoughts or philosophical ideas that imagery painters give to paintings, but also the spirit of a clear era. That is to say, the development of imagery paintings gradually developed based on precisely absorbing humanistic spirit and some technical language in Chinese paintings.

\section{The Meaning and Philosophical Background of "Transcedent"}

There are many explanations for the meaning of "transcedent". Ancient Chinese literati often talked about the two different states of "transcedent" and "mundaneness" to describe their two acts in the world. That is to say, "transcedent" means to give up all the fame and privilege that everyone is chasing, and to choose to escape from the pain of everyday life under unfavorable circumstances in daily life or in the wrong direction of the official career, and to obtain the perception of individual life and the integrity of the spirit in the natural atmosphere. "Mundaneness" is the opposite, the literati obey social etiquette and are engaged in an orderly and active work in order to achieve their social value in real life in the career progress. Since then, Confucianism and Taoism have been used as two different philosophical foundations to achieve agile switching in these two different moods.

Literati painting is the best manifestation of this phenomenon. The literati uses the natural things to express their inner boldness or depression in a clear and elegant manner, and pays more attention to the burst of artistic conception rather than the depiction of objective things. Literati have a significant bearing on the development of freehand painting, and freehand painting advocates similarity. According to $\mathrm{Xu}$ Wei of Ming Dynasty, compared to depicting real objective objects, how to establish the image of the picture and convey the emotion that cannot be directly seen by eyes becomes the primacy of the freehand painting. Through the exploration and contact with nature, painters subjectively summarize and refine the objects they see. While touching nature, the natural charm promotes the painter's own desire for painting. They complement each other and jointly establish a traditional painting text system that is committed to nature but detached from nature.

The formation of such a painting system in China obviously benefits from the conceptual foundation of Taoist ideas. The traditional philosophical concepts behind the imagery paintings mentioned in the previous chapter also benefit from this concept. The Taoist nature that Daoists exalte enable the literati to obtain a strong spiritual support in "transcedent". Traditional Chinese painting emphasizes the affinity and coordination between man and nature, and pursues the "harmony between man and nature". The form of painting does not aim at the performance of objective scenery, but rather pursues the induction of object images and subjective feelings of the subject.

\section{From Different Perspectives, Explain the Sentiment of "Transcedent" in Imagery Paintings}

The performance of imagery painting is a proof of the existence of imagery spirit. In practice, it combines the formal language of Chinese native painting, embodies the picture form of the image, and makes it distinct from other styles of painting and presents a unique language charming. It does not simply imitate nature, but carries out subjective creation on the basis of a profound understanding of natural objects, and skillfully borrows different techniques of language and ideas in Chinese painting. It is a kind of sentimental poetry and a natural poetic mood, which reflects the general "transcedent" of scholars. The following sections will discuss three different perspectives, namely the appearance effect presented of the imagery painting, the commonly selected theme and the temperament presented by the painting.

Explain from the Effects Presented by the Appearance of the Painting - Chinese traditional painting emphasizes "space" which is taken as the carrier to render the artistic conception of beauty. Its intentions are in the absence of ink and are good at seeking "being" from "nothing" and gaining more with fewer painting languages, which does not draw information outside descriptively. Zong 
Baihua referred to it as "a place where life changes and flows", and he believed that it was not easy to reach this realm. Painters must be faithful. "Do not concern to much about secular interestsis" actually consistent with "Being is being and nothing is nothing." and "The heart is empty, God will in the heart and can be unyielding. The movement may disturb God." of Zhuangzi, that is to say, the "space"is not just an external form of painting. It is more closely related to the painter's heart and nature that are not stained by the secular world. It is "transcedent" and extraordinary. Similar to the traditional literati freehand painting, the imagery paintings are also good at extracting and summarizing from the real image. Therefore, the large-scale flat painting or the space are an important way to realize the choice and generalization.

Wu Guanzhong's paintings draw on the expression methods of Chinese paintings through subjective selection and transformation. The object is given a lively and interesting style of charisma, and is expressed in the rhythm of ink and brush. His "space" was echoed with the moving lines and dots, which appeared to be just right. Obviously, Wu Guanzhong implemented the subtraction of colors and shapes in his paintings. When he constructed the spatial sense of the landscape of Southern China with pure color blocks, he also brought in the Chinese ink painting meaning, which was simple, pure, and focused on appearance rather than shape and made the picture produce be more profound implication.

Zhang Dongfeng, a contemporary Chinese painter, often uses a painting method which is similar to the space in his works. However, unlike Wu Guanzhong's, he uses a color that is nearly transparent ib painting. In Mosongtu, which was created in 2009, in terms of screen composition, the composition and color of this work are in some relation with the literati painting. The space left by the painter in this painting is not large, but it is indispensable, which forms a clear color gradation with the dark green and dot-like heavy colors. In another painting which is called Dongfangjibai Series, the gray-and-white sea and sky occupythe main colors of the entire picture.

At the lower part of the sea-sky junction, a thick, heavy black ship comes into view and rests quietly. On the left side of the screen. If there is no such grey, the vast gray may appear pale. If there is no such large piece of gray, then this heavy color may appear isolated and lose its artistic conception. Similarly, in the works of imagery painters, such as Zhao Jiujie and Ou Yang, traces of "space" can also be found, which does not only make the paintings be full of delusions and rhythm, but also permeates "with or without symbiosis" advocated by Taoismin" nothingness" and "lessness", which makes it detach from the secular world and reveals the sentiments of "transcedent" with the harmony and indifferent tone between "being" and "nothing".

Explain from the Selected Theme. There is a strong concept of returning to nature in "transcedent" advocated by Taoism, and the preference of scholars for natural landscape themes is also inextricably linked with this. Taoism advocates returning to nature, thus literati escaped from the difficulties of everyday life and careers and chose the sentiments of "transcedent", which continued for generations in perceiving life and the universe in nature. Similarly, most imagery painters are also keen on landscape subjects. Their works are often closely linked with natural phenomena. They are good at expressing their strong impressions of nature with poetic and lyrical approaches, as well as umanistic feelings. Wu Guanzhong, Zhang Dongfeng, Zhao Jiujie and Ouyang made achievements in this subject matter. Ouyang uses bright colors to express the scenery in her eyes. The paintings display a loose and cheerful charm with bold brushstrokes. The details are gentle and subtle, like the flow of streams. On the contrary, Zhang Qinruo's landscapes are more of a new expressionism and reveal some meditation in some mysterious poetic mood. Compared to the two, Zhao Jiujie's landscape paintings have more of a return to nature and quietness in the heart. These works often give people an illusion and a real sense of presence, making people feel as if they were on the ground. They feel the heart behind this landscape and trees. It is precisely such an elephant-like image that further embodies the unique nature of Chinese philosophy. oneness, spiritual state of forgetfulness. Imagery painters deal with landscape topics from the formal aspects to the spiritual pursuits. Through the attributes of painting materials, they make those seemingly even unstructured lines and color blocks have far-reaching lyricism lyrical sensation in the overall harmonious structure. Both "lawfulness" and "unableness" are the same. The "law" here refers to 
the the law. Taoist nature naturally implies the operating rules and natural laws of the entire universe. Painters sought to improve the natural scenery to become unconscious of the boundary between oneself and the external world, which is the same situation as "transcedent" of literati and scholars.

Explain from the Temperament Presented by the Painting. To a certain extent, imagery painting was influenced by abstractionism and German neo-expressionism, but fundamentally speaking, it was the reproduction of Chinese paintings under the influence of Chinese culture, which is to retransmit China's long-standing culture and philosophy with emerging materials.

In general, the imagery presented by imagery paintings often appears profoundly artistic and extraordinary but not artificial. The works of the painters mentioned in the previous text all exhibit such temperament: relying on the scene, sensation and poetry. Here we have to mention the works of Chinese contemporary painter Hou Jiming. Unlike the most imagery painters, he prefers character themes and is good at merging the relationship between man and landscape. However, his characters are not objectively depicted. The shape does not seem to have a structure or a sense of volume. It is precisely because he gives up the rigid description which makes the characters appear clever in the chaotic but intense colors, which truly realizes dispersion. Although being different from the selection and creation methods of most imagery painters, Hou Jiming's works also give people a profound artistic conception in temperament. This kind of charm is a high degree of refinement and summarization of objective images and conveys the emotion of the self to all things perfectly.

The temperament presented by imagery paintings is different from the formal rationality of the abstractionism and is also different from the rough and agitation of the neo-expressionism. It reveals the ambiguous charm of the expressionism. This ambiguity is not only the blurring or the coloration of the boundary line. The subtle changes between the colors are more of the indescribable mood that presents to viewers. From this point of view, imagery painting still has the extraordinary qualities of literary persons or scholars who have abandoned dogmas, inherent structure or objective things, and had the feeling of being out of the world. And they tried to pursue deeper truths. This is a kind of pure life that painters are difficult to pursue in the secular world.

\section{Conslusion}

The so-called "transcedent" plot has become a significant presence in the literati paintings with a long history. Because the inherent laws of art development are always contextual and inherited, this plot also affects contemporary artistic creation. Imagery painting is a new form of art under the fusion of Eastern and Western cultures. Compared with traditional Chinese paintings, although it has changed in texture, the roots still carry a long-standing internal spirit. This spirit makes the imagery be more vivid. Paying attention to pure painting language and humanistic sentiment, imagery painting is different from the fundamental point of Western local paintings.

\section{References}

[1] Imagery Painting Research. Wang Dongsheng. Culture and Art Press. 2010

[2] The History of Chinese Modern Art.Pan Yaochang. Peking University Press. 2009

[3] Spirit in Art. [Russian] Kandinsky. Translated by Li Zhengwen et al. Chinese People's University Press. 2003

[4] The picture of life - Wu Guanzhong's self-reported history. Wu Guanzhong. Peking University Press. 2011

[5] Art and Visual Perception.[United States] Rudolph Arnheim. Translated by Teng Shouyao. Sichuan People's Publishing House. 2006

[6] Literature Manifesto. Feng Jicai. Culture and Art Press. 2007

[7] Confucian Buddhism Relief Outlook. Wang Wenyuan. Beijing Yanshan Press. 2016

[8] Biography of Zhao Wuji . Zhu Qing. Huashan Literature and Art Publishing House. 1999

[9] The Union of Heaven and Man and Spirit of Chinese Culture. Li Mingjun. Shandong People's Publishing House. 2015

[10] The History of Chinese Painting. Liu Chun. China Youth Publishing House. 2016 